\title{
Lumen
}

Selected Proceedings from the Canadian Society for Eighteenth-Century Studies

\section{L’interaction narrée dans les romans et récits d'Isabelle de Charrière}

\section{Monique Moser-Verrey}

Volume 15, 1996

URI : https://id.erudit.org/iderudit/1012478ar

DOI : https://doi.org/10.7202/1012478ar

Aller au sommaire du numéro

Éditeur(s)

Canadian Society for Eighteenth-Century Studies / Société canadienne d'étude du dix-huitième siècle

ISSN

1209-3696 (imprimé)

1927-8284 (numérique)

Découvrir la revue

Citer cet article

Moser-Verrey, M. (1996). L'interaction narrée dans les romans et récits d'Isabelle de Charrière. Lumen, 15, 135-145. https://doi.org/10.7202/1012478ar 


\section{L'interaction narrée dans les romans et récits d'Isabelle de Charrière}

La communication humaine fait l'objet depuis une trentaine d'années d'enquêtes variées qui tendent toutes à dépasser la primauté accordée traditionnellement au message verbal pour s'intéresser aux conditions de sa production et de sa réception, conditions qui modifient et le sens et les effets de ce message. Dès le début des années quatre-vingt, Yves Winkin résumait l'essentiel des recherches américaines dans ce domaine sous le nom de 'nouvelle communication' et rendait accessible à un public francophone la portée des travaux sur l'interaction humaine réalisés par des anthropologues tels Gregory Bateson, Edward T. Hall et Ray Birdwhistell, mais aussi le psychiatre Paul Watzlawick et le sociologue Erving Goffman. ${ }^{1}$ L'étude des formes de cette interaction, ouverte à un horizon très large incluant la diversité des classes sociales et des cultures, mais aussi la maladie mentale, a permis de voir jusqu'à quel point l'interaction est indissociable de toute communication.

Depuis le début des années quatre-vingt-dix Catherine Kerbrat-Orecchioni a publié une synthèse des notions et des paramètres d'analyse développés dans les sciences humaines. Cette approche est adoptée de plus en plus par la linguistique qui semble être en mutation sous la poussée de ' 1 'interactionnisme. ${ }^{2}$ Du côté des études littéraires, c'est une jeune angliciste allemande qui a dernièrement rendu fructueux le volet non verbal de l'interactionnisme en proposant une théorisation et une histoire du langage du corps tel qu'il est reflété dans la prose romanesque du moyen âge à nos jours. ${ }^{3}$ Il apparaît donc que l'analyse des discours et des textes s'inspire de plus en plus de postulats englobants qui situent ces manifestations verbales dans le cadre de l'interaction humaine en général.

En linguistique, l'objectif de l'analyse des interactions vise à découvrir 'les règles qui fondent le déroulement de divers types $d$ 'interactions verbales. ${ }^{4}$ Il s'agit au fond d'expliciter les partitions invisibles orchestrant toute une variété de matériaux sémiotiques hétérogènes constitutifs de ces interactions. En littérature, par contre, le texte propose une sélection de traits pertinents censée évoquer les types d'interactions que 
l'auteur veut montrer ou raconter. L'analyse de l'interaction des personnages est déjà faite. Ce qui reste à découvrir est cependant la façon dont ces interactions sont représentées et la fonction que leur représentation assume dans l'oeuvre.

Les composantes de base de toute interaction sont le cadre dans lequel elle s'inscrit, les matériaux sémiotiques qu'elle convoque de même que les règles qu'elle suit. Le cadre se décompose à son tour en trois éléments qui sont le contexte spatio-temporel, le nombre et la nature des participants et le but de leur rencontre. ${ }^{5} \mathrm{Si}$ dès lors l'interaction peut être envisagée comme un texte 'produit collectivement dans un contexte déterminé,' elle construit aussi 'la relation de soi et d'autrui dans la communication en face à face. ${ }^{6}$ En elle se manifeste et se modifie la relation interpersonnelle. C'est par ce biais-là que l'approche interactionniste rejoint une caractéristique fondamentale de l'œuvre d'Isabelle de Charrière.

Sur les dix volumes des oeuvres complètes de Mme de Charrière, six volumes sont consacrés à sa correspondance avec plus de 150 personnes différentes. Le face à face que ces correspondances révèlent n'est pas de prime abord celui qu'étudient les chercheurs de la nouvelle communication ou de l'analyse conversationnelle, mais les échanges de lettres construisent aussi des relations interpersonnelles tout en rendant compte souvent $d$ 'interactions en face à face. Dans ses lettres au baron Constant d'Hermenches, Belle de Zuylen appelle, par exemple, les récits de ses interactions avec ses parents des 'histoires." Toute jeune elle se montre déjà très sensible à la manière dont des relations interpersonnelles s'altèrent ou se maintiennent à travers l'interaction et compte beaucoup sur son habileté dans les échanges en face à face pour obtenir ce qu'elle veut de ses parents. Plus tard elle mettra son talent d'observatrice et de manipulatrice à profit dans ses fictions. L'on peut dire sans exagérer qu'elle consacre son oeuvre romanesque et son théâtre à l'étude des relations interpersonnelles, aux rituels d'interaction qui les caractérisent et à la mise en place d'alternatives lui semblant plus justes et plus utiles dans les contextes envisagés.

Cette priorité accordée à la relation infléchit la perspective narrative de notre auteure. La majorité des romans qu'elle a écrits sont partiellement ou entièrement épistolaires et figurent des liens se nouant ou se défaisant dans la perspective restreinte des personnages qui en sont affectés. Dans les deux premiers romans, soit Lettres neuchâteloises et Lettres de Mistriss Henley, les principales épistolières décrivent ellesmêmes leurs interactions avec les êtres qui leur sont proches. Mais dès la rédaction des Lettres écrites de Lausanne on voit apparaître une instance parentale à vocation pédagogique, une mère en l'occurrence, qui lit avec beaucoup de subtilité les interactions les plus secrètes de sa fille avec les 
jeunes gens qu'elle rencontre. Cette mère attentive profite de sa perspicacité et de son expérience pour guider les premiers pas de sa fille dans le monde.

Ce rôle de mentor sera confié ensuite à des abbés, soit l'abbé Des Rois dans Henriette et Richard ou encore l'abbé de la Tour dans Trois Femmes. Une analyse rigoureuse de la perspective narrative adoptée dans ces deux romans montre que d'une part Isabelle de Charrière ne parvient pas à se détacher du recours au narrateur intradiégétique dont le point de vue restreint permet de dramatiser la lecture et l'interprétation des signes non verbaux attachés aux interactions des personnages ${ }^{8}$ et que, $\mathrm{d}$ 'autre part, elle tend à hérö̈ser ces lecteurs pour valider la justesse de leurs interprétations. ${ }^{9}$ Si l'on se souvient qu'avec les Lettres trouvées dans des porte-feuilles d'émigrés, où figure aussi un abbé, ces deux romans forment un tryptique couvrant les trois phases de la Révolution, ${ }^{10}$ on comprend l'intérêt de mettre en scène des directeurs de conscience fiables pouvant aider les jeunes héros à s'orienter dans un monde profondément perturbé.

Il y a tout juste deux cents ans, Isabelle de Charrière entreprenait donc la rédaction du troisième volet de ce tryptique, à savoir le petit roman intitulé Trois Femmes. Qu'il nous serve maintenant à illustrer divers types $\mathrm{d}$ 'interaction visés et construits par notre auteure. En somme, ce roman est resté inachevé, mais ses deux premières parties ont été publiées à Leipzig en traduction allemande dès 1795, puis en français pour le compte du même éditeur en 1798, sous le titre de L'Abbé de la Tour ou Recueil de nouvelles et autres écrits divers. Entre temps des versions moins soignées de la même histoire avaient paru à Londres et à Paris chez d'autres éditeurs pour être reprises plusieurs fois à Paris et en Allemagne au cours de la première décennie du $19 \mathrm{e}$ siècle. $C^{\prime}$ est dire le relatif succès de cette petite oeuvre qui traite un sujet d'actualité, soit l'émigration de la noblesse française, et se situe par rapport au débat philosophique soulevé alors par la notion kantienne du devoir, sans manquer de privilégier le point de vue des femmes sur ces questions brûlantes. Le cadre des interactions figurées est donc l'époque de la Terreur en Allemagne où nobles et prêtres français se réfugient pour sauver leurs vies.

L'histoire des Trois Femmes, telle qu'on la trouve dans l'édition critique des CEuvres complètes d'Isabelle de Charrière, est présentée en trois volets, soit une nouvelle, un récit épistolaire et un roman d'aventure inachevé. Le tout est rattaché par une intrigue très souple ou relâchée dont le but principal est de solliciter, puis de commenter les récits proposés. Il y a enfin, à un dernier niveau, des notes d'éditeur dont l'une, plus développée que les autres, place le tout dans une perspective d'esthétique littéraire. Cette structure n'a bien évidemment rien d'aléatoire, même si les divers narrateurs ont toujours la coquetterie de se 
plaindre de leur style et de leur sujet. Nier tout art, qui pourrait être artifice, et réduire le sujet à ce qui est personnel et accessible, fait sans doute partie du projet tant éthique qu'esthétique réalisé à travers l'oeuvre elle-même. Elle a pour but d'être utile et agréable.

Alix Deguise a consacré une précieuse thèse à ce roman dans laquelle elle privilégie la question du devoir et établit par ailleurs une multitude de rapports entre l'oeuvre de notre auteure et les plus grands écrivains français de même que les plumes les plus marquantes des Lumières européennes. Il y a en effet 'plus de choses qu'il ne semble' dans ce petit roman. ${ }^{11} \mathrm{Et}$, à en croire tout ce à quoi il 'fait penser' selon Alix Deguise, qui mentionne bien des rapports sans pouvoir aller au bout de leurs implications, il reste des découvertes à faire. Parmi les principaux mérites de cette thèse il faut mentionner l'illustration qu'elle offre de la culture très étendue de Madame de Charrière, de même que la preuve qui y est faite de son ouverture aux débats philosophiques les plus récents. On apprend ainsi qu'elle avait sans doute lu et discuté avec Ludwig Ferdinand Huber sa traduction d'un essai de Kant datant de 1793. Il s'agit de 'Das mag in Theorie richtig sein, taugt aber nicht für die Praxis' (Sur le lieu commun: cela est bon en théorie, mais ne vaut rien dans la pratique). ${ }^{12}$

Ce qui me gêne cependant dans la thèse d'Alix Deguise, c'est qu'elle confirme en quelque sorte le lieu commun que Kant s'emploie à réfuter en affirmant que 'tout en ne refusant pas la réalité du devoir kantien [...] elle [Madame de Charrière] a voulu prouver qu'il était presque toujours inapplicable.' Et elle ajoute: 'un concept peut ne pas nécessairement être mis en pratique' (11), ou en d'autres termes: la théorie ne vaut rien dans la pratique. Il me semble qu'Alix Deguise s'est laissée prendre à un faux débat. Madame de Charrière écrit un roman et non pas un essai de philosophie. Contrairement à Kant qui se bat pour des idées et écrit son essai explicitement 'contre' Monsieur Garve, 'contre Hobbes' et 'contre Moses Mendelssohn,' elle ne veut rien prouver par son roman qui se borne à mettre en jeu l'idée du devoir. Elle le dit clairement dans une lettre à Benjamin Constant: 'Le roman ne prétend point prouver l'existence de cette idée, que Kant dit exister'(12).

Le but déclaré des trois histoires proposées par l'abbé de la Tour à la jeune baronne de Berghen est d'occuper agréablement '[sa] sensibilité et [ses] pensées, ne fût-ce qu'un jour ou deux' (41). Aussi ne se soucie-t-elle pas qu'on lui prouve l'existence d'un quelconque impératif catégorique, 'idée universelle et pour ainsi dire innée' (41) du devoir: 'Je n'ai pas trouvé [...] que vos trois Femmes prouvassent quoi que ce soit; mais elles m'ont intéressée, et c'est tout ce que je demandais' (89), dira la jeune baronne à l'abbé. En tant que romancière Isabelle de Charrière ne tient donc pas à avoir raison, mais bien à intéresser le public au sort des 
émigrées françaises. D'ailleurs son histoire se vend par souscription en Angleterre au bénéfice d'une telle émigrée, soit la Comtesse de Montrond. Le roman lui-même propose aussi des solutions viables aux grandes difficultés dans lesquelles se trouvent les émigrées qu'il met en scène. Ce sont des solutions pratiques, mais la façon dont elles sont amenées n'est pas dénuée de fondements théoriques.

Kant pose dès le début de son essai que c'est le jugement qui relie la théorie à la pratique et que toute pratique se rattache à des règles ou des principes même si le praticien n'est pas en mesure de les énoncer. ${ }^{13}$ En tant que romancière Madame de Charrière énonce clairement ses principes et emploie son jugement à mettre ceux-ci en pratique. Elle tient à une écriture utile, pouvant 'instruire et corriger' (135). Or, du point de vue éthique, l'écriture même de Trois Femmes est utile, puisqu'elle génère un revenu que l'auteure emploie à aider une émigrée. Son propos tend par ailleurs à illustrer une affirmation faite par l'abbé de la Tour selon laquelle on peut 'vivre avec tout homme ou toute femme qui a une idée quelconque du devoir' et qui s'applique à remplir ce soi-disant devoir. Les bouleversements dûs à l'émigration suscitent des contextes insolites permettant d'éprouver la validité d'une base de vie commune aussi générale.

Les principes de poétique romanesque d'Isabelle de Charrière se trouvent exposés dans une note d'éditeur s'adressant directement au lecteur. C'est là qu'on trouve, en marge de l'histoire, des conseils pratiques sur la meilleure façon d'écrire des 'leçons vraiment utiles':

Vous qui descendant d'une sorte d'empirée et dédaignant toute vaine exagération, voulez dans une fiction attachante nous faire trouver des leçons vraiment utiles, ayez soin que les caractères de vos personnages une fois tracés et leurs premiers pas faits, il ne leur arrive rien qui pût ne leur pas arriver (134/135).

Cette seule phrase nomme les points essentiels de la théorie qui soustend l'écriture romanesque de Madame de Charrière. Avant tout l'oeuvre doit être utile. Il faut donc que l'histoire soit attachante. Or elle le sera dans la mesure ou chacun pourra y trouver quelque leçon, leçon qui sera rendue péremptoire par 'la pressante logique d'un enchaînement nécessaire de cause à effet' (135). Le texte doit s'offrir comme un champ d'étude à la fois passionnant et convaincant.

L'esthétique bourgeoise prônée par les artistes des Lumières tend à remplacer la question de la beauté par celle de l'intérêt. ${ }^{14}$ Lorsqu'il est question ici d'une 'fiction attachante,' l'éditeur de cette note fait allusion à la notion d'intérêt dans son premier sens selon lequel il s'agit de saisir le public par le biais de ses émotions. Richardson, Voltaire et Laclos, dont les oeuvres sont évoquées dans la suite de cette note, $n$ 'ont pas fait autre 
chose. Mais leurs célèbres oeuvres offrent-elles des leçons 'vraiment utiles' aux hommes et aux femmes? Les incitent-elles à se corriger? L'éditeur qui signe cette note le nie à la suite de Rousseau et soutient que ceux qui seraient intéressés par Clarisse ou Zaïre imagineraient aisément comment échapper au châtiment final, de même que les admiratrices de Madame de Merteuil auraient sans doute déjà eu la petite vérole et ne la craindraient plus. La maladie ou la mort d'un personnage relèvent de l'arbitraire des auteurs et ne peuvent donc pas avoir une valeur absolument dissuasive. Et l'éditeur de poursuivre:

Ce n'est pas qu'il doive, qu'il puisse n'y avoir rien de fortuit dans tout le cours de votre roman mais rien de fortuit n'y doit être décisif (135).

Il ne fait pas de doute pour l'éditeur que la correction des fautes est un élément décisif de toute fiction 'vraiment utile.' Or il n'y a que la raison qui puisse garantir de l'erreur. C'est donc en s'adressant à l'intelligence du public qu'on pourra l'engager à éviter les faux pas. Il ne suffit pas de l'émouvoir par une fiction attachante, encore faut-il offrir à son intelligence matière à réflexion pour qu'il puisse tirer de sa lecture les leçons qui lui conviennent. Il n'y a bien sûr que le déroulement logique des événements racontés qui puisse contraindre la raison à reconnaître quelque leçon que ce soit, et encore...

L'éditeur demande en fin de compte des fictions attachantes et 'ingénieuses' aptes à 'instruire ou corriger le commun des hommes,' mais il ne se flatte pas que de telles fables fassent beaucoup d'effet, puisque les 'sévères et irrécusables' leçons de l'histoire amendent si peu les Princes! En ceci l'attitude de l'éditeur rejoint celle de l'auteur fictif de l'histoire des Trois Femmes. Dès l'abord une autre note de l'éditeur avertit le lecteur que l'on peut raisonnablement reprocher à l'auteur de présenter 'sous toutes sortes de formes le : que sais-je de Montaigne' (42). Il apparaît donc que la stratégie de celui qui veut 'dans une fiction attachante nous faire trouver des leçons vraiment utiles' ne consiste pas à fournir des preuves, mais à soulever des questions facilitant l'émancipation de qui en prend conscience. À ce niveau l'interaction visée est nettement de type pédagogique.

Jusqu'ici j'ai évité d'identifier Madame de Charrière à quelque personnage que ce soit. Il est cependant probable que la théorie proposée par l'éditeur de même que le scepticisme qui réunit cet éditeur à l'abbé de la Tour, auteur supposé du roman, reflètent ses convictions et son sentiment. Il semble bien que Madame de Charrière ait trouvé à cette époque ses aises de narratrice en simulant le point de vue à la fois détaché et engagé d'un directeur de conscience privé de toute autorité. N'y a-t-il pas un certain parallélisme entre les abbés proscrits auxquels elle confie 
ici et ailleurs la voix narrative et la femme philosophe qu'elle se trouve être elle-même? Leur lieu de parole à tous est nécessairement marginal, leur point de vue dégagé et leur engagement pour leurs proches librement choisi.

Si les Lettres écrites de Lausanne peuvent être comprises comme un traité d'éducation de la jeune fille ${ }^{15}$, Trois Femmes se présente comme une réflexion sur la formation de la jeune femme et on peut dire qu'Émilie reprend la carrière de la femme de condition démunie là où Cécile l'avait laissée. En fait la note de l'éditeur qui vient livrer la théorie romanesque de Madame de Charrière amplifie une réflexion de l'abbé narrateur à l'effet que les romans 'gâtent' l'esprit des femmes. Celui-ci vient de raconter combien les romans ennuient Émilie. Elle préfère travailler avec son amie Constance 'Saluste, Tacite et Plutarque' (134). Émilie reproche aux romans un 'style trivial' (133), des personnages sans âme (132), tandis que l'abbé s'en prend à leur morale qu'on dit être sublime, mais qui lui paraît 'plutôt idéale ou qui même n'est plus de la morale ne pouvant s'appliquer à rien' (134). À son idée les leçons de l'héroïsme ne sont guère utiles, même s'il y à des gens qui, contrairement à ce qu'affirme Rousseau dans sa Lettre à d'Alembert, 'se croient obligés d'être tout ce qu'ils admirent' (134).

Le problème réside dans le décalage qu'il y a presque toujours entre le rôle étudié et la 'vérité de la vie' (134). Pour faire plus que 'd'animer par une action intéressante de sages conseils' (135), il apparait que toute fiction doit satisfaire aux exigences suivantes:

1. présenter des personnages attachants dans des situations intéressantes,

2. développer des itinéraires dont l'enchaînement logique est convaincant,

3. proposer une morale commune et adaptée à la vérité de la vie.

En d'autres termes, il faut que les leçons utiles satisfassent les émotions, l'intelligence et le bon sens de chacun. Si un roman peut être considéré comme une théorisation de la vie pratique il faut que le jugement de tout lecteur puisse établir un lien entre l'un et l'autre. Dans ce sens les postulats de Madame de Charrière s'accordent parfaitement avec ceux de Kant.

$C^{\prime}$ est en soignant tout particulièrement la question de l'interaction entre les personnages que notre auteure parvient à introduire la vérité de la vie dans son écriture romanesque. Cette interaction est figurée à plusieurs niveaux du récit dans une recherche constante de justesse. Ainsi le récit primaire expose la motivation qui anime la lectrice des récits à venir. Comme on l'a déjà vu il s'agit de la jeune baronne de Berghen dans le salon de laquelle plusieurs messieurs aux options idéologiques bien définies débattent de la notion du devoir. Mais la jeune 
femme n'a que faire du débat d'idées, ce qu'il lui faut c'est une 'distraction' car elle est très affligée par les maux que la guerre inflige à son pays. L'abbé de la Tour répond à ce besoin en écrivant l'histoire de trois femmes qu'il connaitt, qu'il aime et avec qui il correspond. La vérité de la vie est donc toute proche et la fidélité du rapport qui doit en être fait en quelque sorte garanti par un narrateur témoin particulièrement perspicace.

Les noms de ces deux personnages, soit Berghen (des montagnes) et la Tour, suggèrent une sorte de rencontre au sommet facilitant une vue surplombante sur les destinées dont il sera question. En effet, au début de chacune des trois parties le dialogue entre ces deux personnages reprend, confirmant l'intérêt et même l'admiration que suscite la conduite des trois femmes, conduite qui est cependant loin d'être irréprochable. Le bon coeur de chacune, éprouvé dans des circonstances difficiles, compense au fond les entorses à la vertu. Paradoxalement, la perte d'une certaine pureté vertueuse enrichit des liens d'amour, d'amitié et de reconnaissance (89). Observée dans des situations concrètes, l'idée que se fait chacune de son devoir donne lieu à une discussion moins cérébrale que celle des idéologues proposée au début du roman, car l'émotion y trouve sa juste part.

Tandis que cette interaction 'au sommet' est strictement verbale, les interactions figurées au sein des nouvelles sont attentives à un registre beaucoup plus large d'éléments signifiants. Pour analyser tous les messages vehiculés sans que la parole s'en mêle, il faut 's'y connaître en physionomies' comme le disait Isabelle de Charrière et bien observer ce que l'on appelle aujourd'hui le langage paraverbal et le langage non verbal. En tant que témoin oculaire de plusieurs scènes, l'abbé de la Tour est un interprète privilégié des émotions qui conditionnent le comportement des êtres dont il écrit l'histoire. Mais au-delà de ce qu'il peut avoir vu et entendu, il décrit aussi des scènes plus intimes et secrètes avec une abondance de détails qui ne peuvent être connus que d'un confesseur. La même chose est valable en ce qui concerne les parties du roman confiées à la voix narrative de Constance, la plus expérimentée des trois femmes dont l'abbé est à vrai dire amoureux. Tout se passe comme si ces deux narrateurs, habiles décodeurs des mouvements du coeur humain, renversaient l'attitude éthique du couple Merteuil/Valmont pour montrer comment les scrupules et la délicatesse, quelle que soit la règle qui les dicte, permettent d'entretenir des liaisons heureuses.

Restent les personnages qui sont appelés à former, à cause des désordres de l'émigration, une société nouvelle composée d'éléments hétérogènes. Comment harmoniser les valeurs qu'apportent les émigrées françaises avec celles qui prévalent à Altendorf, petit fief allemand, dont le nom dit très exactement qu'il s'agit d'un village ancien ou, en 
d'autres termes, fidèle à l'Ancien Régime. L'orpheline française de condition, Émilie, y épousera Théobald, le fils du Baron d'Altendorf. Là encore les noms sont emblématiques du projet de l'auteure, d'autant plus qu'une citation de l'Émile de Rousseau apprend à la jeune femme tant l'amour de Théobald que son attachement prioritaire aux 'droits de l'humanité' (57). Tandis qu'Émilie est éduquée par la vie et apprend non sans peines quelle peut être sa place dans la nouvelle société à créer, Théobald s'attelle avec enthousiasme à la tâche d'offrir de l'instruction à ses sujets et de se montrer digne, en Prince éclairé, d'une fonction dont il doit des comptes à Dieu. Dans cette lancée il se promet de publier et distribuer gratuitement un 'Dictionnaire politique, moral et rural' dans lequel on trouve la définition suivante des termes 'obligation ou devoir':

s'explique si différemment par ceux qui exigent et ceux de qui l'on exige, que je ne dirai rien: seulement j'exhorte les deux parties qui auront contracté ensemble, à se consulter et à s'en croire mutuellement à un certain point, sur les obligations respectives (121).

Voilà très exactement la base contractuelle de tout ce qui parvient à se construire dans ce roman de 'métaphysique expérimentale' (116), où rien n'est laissé au hasard.

Parmi les tableaux d'interactions exemplaires proposés tout au long du roman, j'évoquerai pour finir celui qui dévoile les 'contrariétés' que les amants auront à surmonter pour pouvoir s'engager dans une vie commune. Après avoir fait l'éloge des 'jours vraiment heureux' (56) passés par Émilie et Théobald dans l'imprévoyance la plus totale, l'abbé aborde la question des exigences. Il ne sera pas vraiment aisé de s'entendre, Théobald étant 'homme et Allemand; Émilie femme et Française' (56). Émilie pense, par exemple, qu'il n'y a rien de mieux au monde que de vivre à Paris, mais le jeune baron déclare qu'il aimerait mieux servir toute sa vie de tuteur, d'arbitre et de consolateur aux habitants d'Altendorf que de vivre sans utilité pour personne à Paris. Des signes paraverbaux et non verbaux donnent alors à entendre et à voir ce que cette déclaration coûte à Théobald. Et l'abbé de noter que le son de sa voix s'altère à mesure qu'il parle, ce qui décèle un grand trouble. Cet état affectif est ensuite confirmé par une gestualité précipitée et le besoin de mettre plus de distance entre soi et les autres. Théobald sort en effet du salon et même du château (57).

En ce qui concerne le trouble qu'Émilie ressent après avoir lu la citation de l'Émile qui confirme l'amour et les priorités éthiques de Théobald, l'abbé pousse la perspicacité jusqu'à expliciter les pensées les plus secrètes de sa jeune amie: 
je lisais dans ses mouvements, dans sa démarche lente d'abord, puis précipitée: Serait-il vrai? Ces mots me conviendraient-ils? Vous êtes l'arbitre de mon sort! Mais ne vouloir jamais sortir d'ici, et prendre contre moi des précautions, des résolutions si fortes, si décisives! En France les femmes règnent, dit-on. Quelle différence! Ah, mon Dieu! quelle différence (57)!

Les lectures que fait l'abbé des émotions des jeunes amants permettent de voir que les 'contrariétés' sont de taille, mais elles n'empêcheront pas le mariage de l'homme et de la femme, de l'Allemand et de la Française. Même si les choix de Théobald n'ont rien d'excitant pour Emilie, les valeurs qu'il défend forcent le respect. Constance formule sans doute le mieux la raison pour laquelle on peut oser vivre avec cet Allemand:

Oh! la rectitude est bonne. Je n'aurai point de dispute avec Théobald. Je respecte tous les scrupules, les scrupules religieux, les scrupules de l'honneur, enfin tous, ceux même qui n'auraient point de nom, et jusqu'à la soumission à des lois que rien ne sanctionnerait. [...] J'aimerai toujours à voir l'exrême délicatesse se soumettre à des règles qu'elle ne peut définir, et dont elle ne sait pas d'où elles émanent (125).

C'est vraiment l'attention apportée à la justesse et à la qualité des interactions narrées qui constitue la pratique d'écriture par laquelle Isabelle de Charrière entend répondre aux exigences théoriques qu'elle exprime à l'endroit de la forme romanesque.

Ses trois femmes, la servante dévouée, la jeune fille à marier, et la veuve expérimentée, sont attachantes, parce qu'au-delà de leur différences de classe et de fortune elles cherchent le moyen de s'entendre sur ce qu'elles se doivent mutuellement et sur ce qu'elles doivent aux hommes qui les accueillent en terre étrangère, sans pour autant se renier elles-mêmes. C'est la vérité de la vie affective, telle qu'elle est montrée tant au quotidien que dans les grands événements de la vie, qui permet à l'auteure de corriger les préjugés sociaux, les modèles littéraires en vogue et certaines positions philosophiques qui trompent les femmes. Enfin, il est important de voir qu'à l'époque où Kant excluait la femme du cercle des êtres émancipés et doués de raison ${ }^{16}$, Isabelle de Charrière osait mesurer son talent de romancière à la philosophie kantienne et réclamer pour son sexe toute la reconnaissance qu'il mérite. 


\section{Notes}

1 Bateson et all, La Nouvelle Communication, textes recueillis et présentés par Yves Winkin (Paris: Éditions du Seuil, 1981).

2 Catherine Kerbrat-Orecchioni, Les Interactions verbales, tome I (Paris: Armand Colin, 1990) 6. Les tomes II et III du même ouvrage sont parus en 1992 et 1994.

3 Barbara Korte, Körpersprache in der Literatur. Theorie und Geschichte am Beispiel englischer Erzählprosa (Tübingen und Basel: Francke Verlag, 1993).

4 Kerbrat-Orecchioni I, 157.

5 Kerbrat-Orecchioni I, 75 ss.

6 Kerbrat-Orecchioni II, 9.

7 Voir entre autres la lettre au baron Constant d'Hermenches datant du 23-24 juillet 1762.

8 Ce fait a été démontré par Soundouss Ech Cherif El Kettani lors d'une communication prononcée au 62e congrès de l'ACFAS à Montréal le 18 mai 1994 sous le titre : 'Stratégies narratives et comportement non verbal à travers deux romans d'Isabelle de Charrière.'

9 Cette tactique est démontrée dans Anne-Marie Duval, Problématique de la lecture du corps dans Trois femmes $d^{\prime} I$ sabelle de Charrière, mémoire de maîtrise en préparation à l'Université Laval.

10 Isabelle de Charrière, Oeuvres complètes IX (Amsterdam: Van Oorschot; Genève: Slatkine, 1981) 9. Dorénavant, les références à ce volume seront incluses dans le texte. L'orthographe a été modernisée dans toutes les citations pour rendre leur lecture plus aisée.

11 Sainte-Beuve cité par Alix Deguise, Trois Femmes. Le Monde de Madame de Charrière (Genève: Slatkine, 1981) 221.

12 Deguise 9.

13 Immanuel Kant, 'Über den Gemeinspruch: Das mag in Théorie richtig sein, taugt aber nicht für die Praxis,' in Kant, Werke Bd. 9 (Darmstadt: Wissenschaftliche Buchgemeinschaft, 1981) 127-128.

14 voir à ce propos mon article intitulé 'L'Émergence de la notion d'intérêt dans l'esthétique des Lumières,' in L'Homme et la nature VI (1987): 193-207.

15 Claudine Herrmann en parle comme d'un 'véritable traité d'éducation des filles' dans Madame de Charrière, Caliste, Lettres écrites de Lausanne, (Paris: des femmes, 1979) 8 .

16 Immanuel Kant, 'Was ist Aufklärung,' in Berlinische Monatsschrift, Dez. 1784. 